\title{
Brief Discussion on People's Behavior Psychological Demand in Private Courtyard Space
}

\author{
Huaizu Cui ${ }^{1}$, Qingqing $\mathrm{Hu}^{1}$, Fengmiao $\mathrm{He}^{2}$ \\ ${ }^{1}$ Jiangxi Engineering Vocational College, Nanchang, 330025, Jiangxi, China \\ ${ }^{2}$ Nanchang institute of technology, Nanchang, 330013, Jiangxi, China
}

\begin{abstract}
This article mainly describes the method to apply the people's psychological demand in the private courtyard within the scope of environmental psychology. Private courtyard is a place where the activities for house owners are carried out most frequently. The spatial design of private courtyard is in close relation with people's psychological feeling. In the process of design, the environmental psychology will be incorporated into the consideration of people's demand on their physiology, safety, communication, recreation and self-realization, in order to create a humanized, functional and beautiful private courtyard space.
\end{abstract}

Keywords. private courtyard; environmental psychology; behavior psychology

\section{Introduction}

Private courtyard is a place where the activities for house owners are carried out most frequently. The spatial design of private courtyard is in close relation with people's psychological feeling. People that live in the environment will store, understand, organize and reconstruct the incentive from "physical environment" so as to form different feelings and affect their behaviors, or even form an "environmental pressure". In the process of design, the environmental psychology will be incorporated into the consideration of people's demand on their physiology, safety, communication, recreation and self-realization, in order to create a humanized, functional and beautiful private courtyard space.

Environmental psychology, a part of psychology, is a discipline with marginality and comprehensiveness. It's also a science that uses psychological theory and method to make research about the interaction between the environment and the people therein. It mainly studies the optimization of people and environment, and lays emphasis on the target orientation and cycling nature of interaction between people and environment. In brief, it's a "marginal discipline that conducts research on the interaction between environment and psychology of people". It makes analysis on the interrelation and interaction between people and the corresponding environment, mainly to study the interrelation and interaction between environment and people's behavior psychology. It tends to study people's certain psychological activities and behavior features in a certain environment. This article focuses on the introduction of people's behavior psychological demand which shall be noted in the design of private courtyard space.

\section{Relation of behavior psychology and space}


Psychological refers to the mentality and emotion of people, and can be divided into three aspects, namely cognitive process, emotional process and willed process, including people's emotion and feeling $^{[1]}$. Behavior refers to people's various daily activities, or their external activities enough to indicate people's mind, quality, psychology and other aspects.

Behavior is a response of psychology which otherwise controls the behavior. Behavior is to meet people's demand ${ }^{[2]}$. People's behavior psychology is a reflection of their various basic demands in the environment.

Space, if not interacted with people's behavior, won't have any practical meaning, because it's just a functional carrier; People's behavior, if not having a spatial environment as its background or a certain atmosphere condition, will be unlikely to happen. The incorporation of space and human behavior comprises a place for human that meets various behavioral demand thereof. Only in this way will a space have an actually practical meaning.

People relation shall be considered in the private courtyard space. There are mainly two kinds of people's activities in the private courtyard space: psychological and behavioral activity. Psychological activity refers to people's cognition and understanding about environment, and behavior activity refers to people's motion and behavior in the environmental space. The interaction of psychology and behavior will affect the way people use the space, and further influence the value expression of space $^{[3]}$.

\section{People's behavior psychological demand in private courtyard space}

The residential behavior psychology of residents comprises a special demand on private courtyard space. People's behavior psychological demand in private courtyard space is a reflection of their various basic demands in the private courtyard space.

There are many researches on people's demand conditions, among which the most influential one is the demand hierarchy progression theory proposed by Maslow, a famous American humanistic psychologist. In his book, Theory of Human Motivation, he divided the human demands into five hierarchies: physiological demand (coziness of survival and health), safety demand (protectiveness, privacy, fear, etc.), belonging and loving demand (love, sex, sentiment, friendship and independence), respect demand (reputation, authority, appraise, success) and selfrealization demand. (as shown in Figure 1). People will first pursue the demands in lower hierarchy, and they will further pursue the demands in higher hierarchy only after the satisfaction of ones in lower hierarchy. The demands in different hierarchies are reflected in the space through behavior psychology of residents, which indicates various behavior psychological demands of residents on the space. ${ }^{[3]} \mathrm{A}$ desirable private courtyard space shall meet various demands of the residents (as shown in Figure 2).

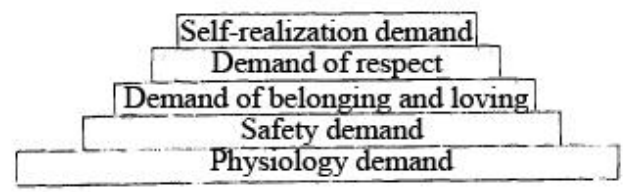

Figure 1. Demand hierarchy theory of Maslow

\begin{tabular}{|c|c|}
\hline People's basic demands & Behavior psychological demand on the space \\
\hline Physiology demand & Coziness \\
\hline Safety demand & Safety sense, privacy, territoriality \\
\hline Demand of belonging and loving & Belonging sense \\
\hline Demand of respect & Territoriality \\
\hline Elf-realization demand & Self-realization (involvement, independence) \\
\hline
\end{tabular}

Figure 2. Correspondence relation of people's basic demands and space behavior psychological demands 


\subsection{Demand on coziness}

The coziness is a common target for people's psychology, and one of people's basic physiological demands. It is composed of multiple hierarchies and factors. It includes functional convenience, physiological harmony and psychological joy and happiness. Its content mainly encompasses sufficient area, perfect facility, desirable physical conditions (sound insulation, heat insulation, warm keeping, lighting, ventilating condition, etc.) and other objective material demands. Those objective environments centering our humans give out signals to stimulate people's sense organs, and further induce changes in psychological coziness of residents. The psychological coziness is closely related to physiological demands. There will definitely be psychological non-coziness if the physiological demands are not satisfied. However, a residential environment that meets the basic physiological demands is not necessarily a cozy environment for residents, because the people may adjust their behavior in some way to adapt to the surroundings. So, a cozy environment shall have a perfect answer to people's residential physiological demand.

\subsection{Safety and privacy demand}

Safety demand is one of the basic demands for human to survive in this world. People's safety demand is reflected mainly through the demand on safety and privacy. People's initial purpose to construct housing was to hide from wind, rain and weather change, as well as to protect themselves from the attack of beasts and other tribes, which come down to requirement for safety. From what is mentioned above, we can see the importance of safety sense in the residential life. With the development of human life, the residential types are gradually differentiated, and there is a variety of private courtyards related thereto. While the feature to keep men's security is never changed.

According to the theory of environmental psychology, an individual or group of people expect a freedom in controlling and selecting other people or external environment for information exchange, and the privacy demand is actually the demand of that control mechanism and function. It's one of people's basic behavior psychology demands. Privacy is conducive to establishing the sense of self-unification. Privacy involves a dynamic process. People may keep a safe distance by adjusting their closeness to other people. ${ }^{[4]}$ So a certain privacy needs to be kept in the private courtyard. This demand is reflected in different ways in different cultures: British American takes the lawn in front of his residence as a symbol to indicate the outdoor private space and group unification; Danish usually grows beech hedges of one people's height in front of residence, similar to the hints of lawn and fence; In Chinese traditional culture, the residential building and private courtyard form an indivisible whole. Taking the quadrangle in Beijing as an example, it is enclosed by rooms to form a private courtyard closed from outside and opening to inside. The door therein faces the gable of wing-rooms through passages. It is impossible to see the inside of private courtyard of either a small or a large quadrangle.

\subsection{Territoriality Demand}

A clear spatial territoriality is an important way to meet people's "demand on respect". The concept of "territory" originates from ethology, and it refers to an area protected from other organizational members. It is found from the observance of psychologists and socialists that people's territoriality is an instinct behavior, but influenced by cultural background. The territoriality, an ability and sense for an individual or group to control a certain place or object, is formed when the owner of a territory identifies this territory and express it in some way. It is reflected in behavior that people can use the space in the way they like, or change the space in nature to reflect their features. To be more specific, this expression in the actual private courtyard environment establishes a symbol of territory, including the actual and symbolic mark, such as keeping the private courtyard environment clean, beautifying the private courtyard, fences, walls 
and other borders of territory. The establishment of territory may increase people's sense of control to the environment, and enable them to control the behavior of other people to some extent. If the residents see the private courtyard space as their own territory, they will form a common sense of territory and responsibility inside the private courtyard, strengthen the monitor and control of the environment and discover unusual situations in a timely manner; The ownership of the territory will also intensify the determination and capacity to defend the territory in order to fight against stronger aggressors.

\subsection{Demand of belonging sense}

Demand of loving and belonging sense is a psychological demand in higher hierarchy, with more tendency to mental demand. Belonging sense refers to the love, care, attachment, identity and other psychological senses of residents for their own territory and people, such as the positive sense for the position of private courtyard, markers, etc., the sense of pride and other special emotions induced therefrom. In the composition of social group of people, the people's belonging sense is a most basic emotion and demand. As said by Alexander: "People need an identifiable special unit for their belonging". People will get the sense of belonging to meet the psychological demand "loving and belonging sense" in an easiest way by joyful and natural communication in a familiar, identified and cozy environment.

Communication is an important condition for residents to realize their belonging sense. The psychological barrier will be removed and the belonging sense will be intensified in a clear territory space. The clear spatial division of private courtyard is helpful to the occurrence of communicative activities. The shape, position, dimension and other aspects of different communicative spaces shall fully consider people's place psychology and create a spatial environment that satisfies people's psychological demand in order for them to be willing to communicate with each other in this joyful and natural atmosphere so that they can meet their communicative demand. The multiple activity facilities that satisfy various behavior demands of residents offer an opportunity for the people in this space. ${ }^{[5]}$

Besides mental factors, the material factors are also very important to satisfy the belonging sense. The identifiability of residence is closely related to the formation of resident's belonging sense. The research on the environmental psychology indicates that if there's a clear identifiability in the space of residential environment, the residents will tend to generate a strong sense of identity for the space and realize the mental and psychological attachment relation of themselves with the residential environment, leading to the formation of a belonging sense to the residential space. Therefore, it is very important to set up a private courtyard environment with desirable sense of belonging.

Marginal elements are the primary enclosing elements for private courtyard space. The colors and textures of their dimensions, types, outlines and external materials directly reflect the external spatial image of private courtyard, and meanwhile create an atmosphere of private courtyard space jointly with independent elements to influence the psychological feelings of residents. There are many complex reasons why the private courtyard landscapes in different territories have different styles, but one of the important causes is that all of them desirably comply with the psychological identity, and are greatly accepted and loved, by local residents.

\subsection{Self-realization demand}

The self-realization demand, a mental demand in the high hierarchy for human, is reflected in the reconstruction and creation of environmental dynamics, including the changes by artificial and natural factors to enable the courtyard space to meet the resident's high hierarchical demand on recreation, entertainment, communication and spiritual benefit. All people are hoping to form a private courtyard environment according to their own thought to meet the goal of self-realization. In the landscape design of private courtyard space, the resident is not only a designer, but also a 
user. They have certain involvement and strong independence in their own private courtyard. They can design as their own conception, demand and preference. Having independent right of use, they may also change the landscape of their private courtyard at any time to adapt to the change in family population and lifestyle, as well as have a featured and beautiful style of private courtyard with certain identifiability.

\section{Conclusion}

The environmental psychology is seen to be applied everywhere in the design of private courtyard space, so we can just analyze and discuss a tiny part of it. But the courtyard design shall be people oriented, respect people's behavior psychological demand and create a pleasant design of private courtyard suitable for people's use.

\section{References}

1. Xu, L.Q., Environmental Psychology [M]. Shanghai: Tongji University Press, 2002.

2. Ma, T.D., Environmental Psychology and Psychological Environment [M]. Tianjin: Tianjin Science and Technology Press, 1996.

3. Zhao, C.C., Environmental Psychology [M]. Lanzhou: Gansu People's Publishing House, 1990.

4. Wang, Y.J., Fundamentals of Social Psychology [M].Beijing: China Renmin University Press, 2001.

5. Han, Y.X., Return to Spiritual Homeland--Reconstruction of Featured Space of Chinese Traditional Residential Environment [D]. Master Thesis from Dalian University of Technology. 2004. 